\title{
British Stomach Cancer Group
}

The 1994 meeting of the British Stomach Cancer Group was held on 17 June 1994 at the University of Liverpool, Royal Liverpool Hospital. The abstracts presented at the meeting are printed below. ${ }^{\star}$
Comparison of the molecular genetics of stomach cancers in Britain and Japan: cErbB2 and p53

P MCCULLOCH, A OCHIAI, J O'DOWD, J NASH, M SASAKO, S HIROHASHI (Departments of Surgery and Pathology, University of Liverpool and National Cancer Center Hospital, Tokyo The incidence, stage at diagnosis, and curability of gastric cancer differ markedly between Japan and Western countries. The genetic and environmental influences on gastric carcinogenesis are very different in the two populations, and some Western authors have postulated a less aggressive type of cancer in Japan. We sought evidence of differences in tumour biology by comparing the incidence of p53 and c-erbB2 gene abnormalities in British and Japanese gastric cancer. Eighty five British cases were carefully matched for $T$ stage and tumour grade with 89 contemporary Japanese cases. Archival paraffin sections were microwaved and immunohistochemically stained by 2 stage alkaline phosphatase/anti-alkaline phosphatase technique. The results are shown in the Table.

$\begin{array}{llll} & & \text { British } & \text { Fapanese } \\ & + & 46 & 46 \\ \text { p53 } & \pm & 39 & 43 \\ \text { c-erbB2 } & \pm & 26 & 28 \\ & - & 59 & 61\end{array}$

Diffuse cancers from each centre were less commonly positive for either antigen than intestinal cancers.

British and Japanese tumours matched for stage and grade show similar frequencies of p53 and c-erbB2 abnormalities. These results provide no support for the idea of different forms of gastric cancer in East and West.

Different levels of p53 mutation in proximal and distal gastric cancers

N J KEELING, J I LIVINGSTONE, M I FILIPE†, C WASTELL (Department of Academic Surgery Chelsea and Westminster Hospital, 369 Fulham Road, London, † Department of Pathology, Guy's Hospital, London Bridge, London) Mutant p53 protein expression has been reported in up to $60 \%$ of solid human cancers. Evidence suggests that normal p53 mediates the cellular response to DNA damage by monitoring the fidelity of cell replication. Its exact mode of action remains unclear. Most reports suggest that p53 mutations are associated with advanced disease in gastric cancer.

Our aim was to determine whether a difference in p53 expression exists between

*The abstracts Oestrogen receptors in gastric cancer international differences (T Ismail, P Baker, C Hale, $\mathrm{J}$ W L Fielding, M T Hallissey) and The dyspepsia study: the impact on survival in gastric cancer (M T Hallissey, J Harrison, L Ward, W H Allum, A Jewkes, J W L Fielding) have previously been presented at the British Association of Surgical Oncology and will be published as abstracts in the European fournal of Surgical Oncology. The abstracts European fournal of Surgical Oncolo
are therefore not included here. proximal gastric cancer and distal gastric cancer in view of their different epidemiology, prognosis, and possible aetiology.

Formalin fixed specimens of 80 consecutive human tumours resected in our unit were studied. Satisfactory tissue and staining was obtained in 69 tumours using mouse antihuman antibody DO-7 with microwave incubation.

\begin{tabular}{|c|c|c|c|c|}
\hline & Number & $\begin{array}{l}\text { p53 } \\
\text { Positive }\end{array}$ & $\begin{array}{l}\text { p53 } \\
\text { Negative }\end{array}$ & $\begin{array}{l}\% \\
\text { Positive }\end{array}$ \\
\hline ximal & & 15 & 12 & 55.6 \\
\hline ta & 38 & 11 & 27 & 28.9 \\
\hline $\begin{array}{l}\text { iffuse } \\
\text { otal }\end{array}$ & $\begin{array}{l}4 \\
69\end{array}$ & $\begin{array}{l}1 \\
27\end{array}$ & $\begin{array}{l}3 \\
42\end{array}$ & $\begin{array}{l}25 \\
39 \cdot 1\end{array}$ \\
\hline
\end{tabular}

A significant difference in p53 expression was seen between proximal and distal stomach $\left(\chi^{2}=0.05\right)$. These results suggest that the cell biology of proximal and distal gastric cancers may be different.

Expression of growth factors in precursor lesions to gastric carcinoma

M I FILIPE ${ }^{1}, M_{\text {OSBORNE }}^{1}$, M J BRITO ${ }^{1}$, J LINEHAN $^{1}$, E SANIDAS ${ }^{1}$, J JANKOWSKI ${ }^{2}$ (Department of Histopathology, UMDS, Guy's Hospital, London $^{1}$, ICRF, RPMS, Du Cane Road, London $^{2}$ ) Epidermal growth factor (EGF), its related peptide transforming growth factor $(\mathrm{TGF} \alpha)$, and their common receptor (EGFR) have been implicated in the control of cell proliferation and differention in the gastrointestinal epithelium and may play an important part in gastric carcinogenesis.

We assessed the immunohistochemical expression and topographic distribution of these peptides and western bolt analysis in the precursor lesions to gastric carcinoma compared with their counterparts in non-cancer tissue.

We observed: (i) Increased and extended expression of TGF $\alpha$ in normal mucosa and hyperplasia in carcinoma fields compared with non-cancer controls. (ii) Increased expression of EGFR in intestinal metaplasia (IM) from carcinoma fields than in controls. (iii) EGF expression was not detected in normal mucosa and only weakly in IM. (iv) Coexpression of TGF $\alpha / E G F R$ and EGF/EGFR was higher in IM in carcinoma fields than in non-cancer controls. We conclude that altered expression of TGF $\alpha / E G F R$ follows a chronology of events to gastric carcinogenesis. In this regard increased expression of TGF $\alpha$ is a very early event, which is subsequently followed up by regulation of EGFR and this has important biological and clinical implications.

Gastrin receptor localisation in normal and neoplastic gastric mucosa

R E SYKES ${ }^{1}$, G ROBINSON ${ }^{1}$, S A WATSON ${ }^{2}$, (Department of Pathology ${ }^{1}$, Department of Surgery ${ }^{2}$, University of Nottingham) Numerous in vitro studies have shown that the polypeptide hormone, gastrin has a trophic effect on the gastric mucosa. However, little is known about the cellular localisation of gastrin receptors in the stomach.

The availability of $2 \mathrm{C} 1$, a monoclonal antibody directed against the canine parietal cell receptor, has permitted the immunocytochemical localisation of gastrin receptors in the human gastric mucosa to be undertaken in formalin fixed, paraffin embedded tissue.

Gastric adenocarcinomas and adjacent normal mucosa were examined in 25 patients and the gastrin receptor localised using the avidin biotin complex (ABC) technique. Immunoreactivity in the normal mucosa was evident in the cardiac, body, and pyloric regions, with the strongest activity being present in the parietal cells. The mucosa in the gastric adenocarcinomas stained uniformly and diffusely, with the strongest staining being associated with poorly differentiated adenocarcinomas.

The ability to localise by immunocytochemistry gastrin receptor activity in formalin fixed, paraffin embedded material may prove useful as a pre-clinical screen for patients undergoing therapy with anti-gastric agents.

Laparoscopic assessment of resectability in gastric cancer

R AUSTIN, M C WINSLET (University Department of Surgery, Royal Free Hospital, Pond St, London) The use of preoperative laparoscopic assessment, in addition to computed tomography (CT), has been advocated in gastric cancer to identify patients who would benefit from preoperative chemotherapy. The relative accuracy of CT and laparoscopy compared with operative and histological assessment is poorly defined.

Twelve patients $(\mathrm{m}=9, \mathrm{f}=3$; mean age 62 (47-80) years) with gastric carcinoma (distal $=7$, proximal $=5$ ) underwent preoperative CT and laparoscopy with visualisation of the lesser sac before resection (partial $=7$, total=5). Results were compared with operative and histological assessment. Five patients had distal involvement (hepatic $=2$, peritoneal $=3$, ascites $=3$ ); 11 had local involvement $\quad$ (pancreatic $=3, \quad$ retroperitoneal $=5$, nodal $=11$ ).

\begin{tabular}{lcccccc} 
& CT & & \multicolumn{4}{c}{ Laparoscopy } \\
\% & Local & Distal & Overall & \multicolumn{1}{c}{ Local } & Distal & Overall \\
Sensitivity & 55 & 60 & 36 & 36 & 60 & 27 \\
Specificity & 100 & 100 & 100 & 100 & 100 & 100 \\
PPV & 100 & 100 & 100 & 100 & 100 & 100 \\
NPV & 17 & 78 & 13 & 13 & 64 & 11 \\
Productive & & & & & & \\
$\quad$ efficiency & 58 & 83 & 42 & 42 & 83 & 25
\end{tabular}

Preoperative laparoscopy has little advantage over CT apart from identifying distal micrometastisis. Both techniques, however, have a poor predictive efficiency and indicate the need for urgent evaluation of potentially more accurate techniques such as endoluminal ultrasound. 
CT scanning and carcinoma of the gastro-oesophageal junction

D J CORLESS ${ }^{\star}$, N DIMANI $\dagger, T$ EL SAYED $\dagger$, J MEYRICK THOMAS (Departments of Surgery and Radiologyt, Watford General Hospital, Vicarage Road, Watford, Herts) The increased incidence of cancer of the gastric cardia and gastro-oesophageal junction (GOJ) is unexplained. These tumours and those of the distal oesophagus present a greater surgical challenge than distal cancers. Accurate information about the tumour is necessary to not deny surgery to those who may benefit and to suggest other treatment modalities if the tumour is extensive. Computed tomography provides precise information about the thoracic oesophagus where fixed adjacent structures provide reference. At the GOJ the anatomy is variable and mobile and scanning less accurate.

The aim of this study was to evaluate barium sulphate suspension (EsophoCat) as the oral contrast medium in preoperative CT for tumours of the GOJ and distal oesophagus. Fourteen patients were available to compare CT with the surgical and pathological findings.

$\begin{array}{llrll}\text { Site } & \begin{array}{c}\text { True } \\ +v e\end{array} & \begin{array}{c}\text { True } \\ -v e\end{array} & \begin{array}{c}\text { False } \\ +v e\end{array} & \begin{array}{l}\text { False } \\ -v e\end{array} \\ \begin{array}{l}\text { Aorta; } \\ \text { pericardium; lung }\end{array} & 1 & 13 & 0 & 0 \\ \begin{array}{l}\text { Local spread } \\ \text { Gastro-hepatic }\end{array} & 3 & 5 & 0 & 6 \\ \quad \text { nodes } & 1 & 4 & 0 & 9 \\ \begin{array}{l}\text { Mediastinal nodes } \\ \text { Liver }\end{array} & 0 & 13 & 0 & 1 \\ & 2 & 9 & 1 & 2 \\ \text { Site } & \text { Sensitivity } & \text { Specificity } & \text { Accuracy } \\ \begin{array}{l}\text { Aorta; } \\ \text { pericardium; lung }\end{array} & 100 \% & 100 \% & 100 \% \\ \begin{array}{l}\text { Local spread } \\ \text { Gastro-hepatic }\end{array} & 33 \% & 100 \% & 57 \% \\ \begin{array}{c}\text { nodes } \\ \text { Mediastinal nodes } \\ \text { Liver }\end{array} & \begin{aligned} 10 \% \\ 0 \%\end{aligned} & 100 \% & 35 \% \\ & 50 \% & 90 \% & 93 \% \\ & & & 79 \%\end{array}$

These results confer with other series with a low sensitivity and high specificity for local spread and nodal involvement. Advanced and very early cases are accurately staged. The majority between these extremes present greatest difficulty. This medium provides excellent coating of the oesophageal lumen permitting accurate assessment of wall thickness and tumour. Scanning will exclude gross spread if palliative surgery is planned but if more accurate information about the tumour is required other modalities such as endoluminal ultrasound should be considered.

\section{'Curative' gastrectomy from gastric carcinoma - $\mathbf{R} 1$ versus $\mathbf{R 2}$}

N T WELCH, J W L FIELDING (The Queen Elizabeth Hospital, Edgbaston, Birmingham) Survival after gastrectomy for carcinoma may be increased by a radical lymphadenectomy (R2 gastrectomy). This supposition is examined in 97 consecutive patients who have undergone curative R1 (53 patients) or R2 (44 patients) gastrectomy for Stage I-III disease.

The 30 day postoperative mortality was $3.8 \%$ for R1 (two deaths) and $11 \cdot 4 \%$ for R2 (five deaths). The excess deaths after R2 were due to pancreatitis (two deaths) and gastric remnant infarction (two deaths). Actuarial five year survival for R1 and R2 was $35.5 \%$ and $39.8 \%$ respectively but at eight years the difference was more obvious at $20 \%$ and $39.8 \%$ respectively.
The survival advantage for $\mathrm{R} 2$ gastrectomy is only seen after five years, but there is an associated greater operative mortality. Operative mortality may be reduced by avoiding routing distal pancreatectomy, merely performing a lymphadenectomy.

\section{Short term morbidity and mortality in the Dutch R1-R2 trial}

J J BONENKAMP 1 , I SONGUN ${ }^{1}$, J HERMANS ${ }^{2}$, C J H van de Velde ${ }^{1}$ for the Dutch Gastric Cancer Group (DGCG) (University Hospital Leiden, Department of Surgery ${ }^{1}$, Department of Medical Statistics ${ }^{2}$ ) In Western patients gastric cancer has a bad prognosis, whereas in Japan superior results are claimed after extended lymphadenectomy (R2 resection). Researchers in specialised Western centres have started to ascribe improved results to radical surgical therapy as well. In the Netherlands in the period between August 1989 and July 1993 a prospective randomised trial was done to evaluate the role of the $\mathrm{R} 2$ resection in the treatment of gastric cancer. First data of this trial, the short term morbidity, and operative death rates, are discussed here.

In the case of a histologically proved adenocarcinoma of the stomach, patients were assigned $\mathrm{R} 1$ or $\mathrm{R} 2$ by preoperative randomisation. The operation was attended by a consultant surgeon, who assessed whether a curative resection was possible. In that case an $R 1$ or $R 2$ resection was performed according to the rules of the 'Japanese Research Society for the Study of Gastric Cancer' (JRSGC). In non-curable patients the surgeon performed a palliative operation according to his own discretion and irrespective of the randomisation.

Seventy eight participating institutions randomised 1078 patients (539 R1 and 539 R2). Of these, $996(92 \%)$ fulfilled the inclusion criteria. Seven hundred and eleven patients had a curative resection (380 Rl and $331 \mathrm{R} 2$ ) and 285 patients (29\%) had a palliative procedure ( $53 \%$ palliative resection, $19 \%$ bypass, and $27 \%$ laparotomy only). In the whole group (curative and noncurative) complications occurred in $31 \%$ (R1 25\%, R2 38\%) and reoperation was required in $10 \%$ ( $\mathrm{R} 17 \%, \mathrm{R} 214 \%$ ). R2 patients had a longer postoperative hospital stay (R1 17.9 days, $R 222.4$ days) and higher operative death rate (R1 5\%, R2 10\%). In the curative group the differences were still present: complications $25 \%$ versus $43 \%$, reoperations $8 \%$ versus $16 \%$, hospital stay 18.0 versus 24.7 days and postoperative death $4 \%$ versus $10 \%$ for $\mathrm{R} 1$ and $\mathrm{R} 2$ respectively. These differences are all significant.

In contrast with the results of retrospective studies, R2 resection for gastric cancer does give significantly more complications and a longer mean hospital stay and the operative mortality is more than twofold higher compared with the $\mathrm{R} 1$ resection. These factors will have to be weighed against the expected improvement of the survival. Final survival data will not be available before 1995. Until then the place of the R2 resection in the treatment of gastric cancer remains uncertain. Therefore it is recommended that more attention is given to multimodality treatments in the search to improve the prognosis of gastric cancer. Therefore the DGCG started a new trial (POCOM) in which the effect of pre- operative chemotherapy for gastric cancer is evaluated.

Japanese style gastric resection - viable in the UK?

J I LIVINGSTONE (Department of Surgery, Kingston Hospital, Galsworthy Road, Surrey) The Japanese approach to gastric cancer surgery is commonly hailed as the 'gold standard' to which European surgeons should aspire. Is such a comparison valid? After a visit to the National Cancer Center in Tokyo, the author notes the following advantages bestowed upon the Japanese surgeon. (1) Early diagnosis - screening and health education so that $50 \%$ of cases are early cancers. (2) Comprehensive preoperative endoscopy and radiology, such that both distant and local tumour extent are accurately defined. (3) Multi-disciplinary decisions on treatment. (4) Optimal surgical facilities specialist theatre staff and equipment, unlimited operating time. (5) Dedicated pathology. (6) Specialist postoperative care with aggressive management of complications.

Without simultaneous development of associated specialties, it is unlikely that UK surgeons will be able to exploit the full benefits of radical surgery.

Can prescribing omeprazole delay the diagnosis of gastric malignancy?

N HAYES, S A RAIMES, S M GRIFFIN (Department of Surgery, Newcastle General Hospital, Newcastle upon Tyne) The proton pump inhibitor omeprazole achieves faster healing rates and symptom relief from peptic ulcer disease than the $\mathrm{H}_{2}$ antagonists. It is recognised that $\mathrm{H}_{2}$ antagonists can relieve symptoms and heal early superficial lesions in patients with gastric cancer. This phenomenon has not yet been reported in patients who have taken omeprazole.

During a 12 month period, we saw five patients with gastric neoplasm, in whom complete relief of symptoms was obtained after treatment with omeprazole. In each case there was evidence of 'healing' of the lesion on endoscopy. In four of these patients, the omeprazole was prescribed before a histological diagnosis was made and in those, the interval to diagnosis ranged from 6-50 weeks. Four patients had adenocarcinoma, three had stage I disease and one had a stage II tumour. The fifth patient had a stage II $E$ nonHodgkin's B cell lymphoma. All five patients underwent surgery, they suffered no complications and all are currently well.

This is the first series of cases recorded in which omeprazole has abolished the symptoms and signs of gastric neoplasms. Our unit's aggressive biopsy practice resulted in the correct diagnosis of these 'healed' lesions. Had any further delay occurred, these cancers could have been missed with disastrous consequences. If 'dyspeptic' patients are offered potent antisecretory drugs without investigation, those with gastric tumours may have a temporary improvement in symptoms. It is highly likely that some of these patients will be denied the chance of early treatment because of avoidable delays in diagnosis. We conclude that the practice of prescribing potent antisecretory drugs for 'dyspeptic' patients over 40 years of age without first obtaining an accurate diagnosis is to be condemned. 
Gastric cancer and Helicobacter pylori infection

D J CORLESS, J I LIVINGSTONE, C WASTELL (Academic Surgery, Chelsea and Westminster Hospital, 369 Fulham Road, London) The paradoxical rise in proximal gastric cancer despite an overall decline in the disease is unexplained. Helicobacter pylori $(\mathrm{Hp})$ has been shown to be associated with distal gastric cancer but not with tumours of the gastrooesophageal junction and cardia. The aim of this study was to identify any differences in the prevalence of infection with $\mathrm{Hp}$ in different patient groups with gastric cancer.

Original tissue blocks of cases of gastric cancer between 1980 and 1990 were resectioned and examined. All areas of gastric mucosa were stained with a polyclonal antiHelicobacter stain (Dako) using a standard three layer immunohistochemical technique. Tumour site, age, sex, and degree of tumour spread (localised, regional spread, and disseminated) were also recorded.

There were 51 men and 30 women giving a ratio of $1 \cdot 7: 1$. The mean age at diagnosis was
65.4 years with a range of 34 to 86 . Hp was identified in 52 cases $(64 \cdot 2 \%)$. When analysed with respect to tumour site more proximal cases, 23 of $29(79 \%)$ were positive compared with distal cases 24 of $45(53 \%$; $\left.x^{2} \quad \mathrm{p}<0.05\right)$. There was no association between $\mathrm{Hp}$ and age, sex or degree of tumour spread.

These results are in broad agreement with other series with regard to the total number of positive cases but suggest that $\mathrm{Hp}$ may also be involved in the aetiology of proximal cancers. 